\title{
NEUROMIOTONÍA OCULAR
}

\section{OCULAR NEUROMYOTONIA}

\author{
ASENSIO-SÁNCHEZ VM ${ }^{1}$, MORALES-GÓMEZ I ${ }^{2}$, RODRÍGUEZ-VACA I ${ }^{1}$
}

\begin{abstract}
RESUMEN
Caso clínico: Se describe un paciente de 58 años, sin antecedentes de radioterapia, que presenta episodios de diplopía después de una mirada lateral y mantenida de 30-50 segundos de duración. Durante el episodio desarrolla una exotropía de gran ángulo en el ojo izquierdo retornando a la normalidad después de 50 segundos. El paciente se encontraba asintomático entre los episodios de diplopía. El paciente fue tratado con carbamacepina con resolución parcial de los síntomas.

Discusión: La neuromiotonía ocular debe ser considerada cuando se evalúa un paciente con diplopía intermitente.
\end{abstract}

Palabras clave: Neuromiotonía ocular, diplopía intermitente, enfermedad intracraneal, carbamacepina.

\begin{abstract}
Case report: We describe a 58-year-old man, without history of radiation therapy, who presented with transient episodic diplopia following sustained lateral gaze lasting around 30-50 seconds. He developed a large angle exotropia of the left eye that gradually returned to normal after 50 seconds. Between episodes he was asymptomatic. Partial resolution of his symptoms was obtained after treatment with carbamazepine.
\end{abstract}

Discussion: Neuromyotonia must be considered when evaluating a patient with intermittent diplopia (Arch Soc Esp Oftalmol 2008; 83: 673-674).

Key words: Ocular neuromyotonia, intermittent diplopia, intracranial disease, carbamazepine.

\section{INTRODUCCIÓN}

La neuromiotonía ocular (NO) es una enfermedad rara de la motilidad ocular caracterizada por la contracción involuntaria de uno o varios músculos oculares (1). Generalmente es secundaria a otros procesos asociados, aunque se han descrito casos idiopáticos $(2,3)$. En este trabajo se presenta un caso típico de neuromiotonía ocular calificado como idiopático.

\section{CASO CLÍNICO}

Varón de 58 años, sin antecedentes de interés, que acude al servicio de urgencias enviado por su médico de atención primaria para descartar «patología retiniana» por diplopía intermitente de un año de evolución pero más frecuente en la última semana. De profesión camionero, refiere entre 10 a 15 episodios al día de diplopía horizontal, durante 30-50 segundos sólo cuando mira por el retrovisor izquier-

Recibido: 24/9/07. Aceptado: 13/10/08.

Hospital General Medina del Campo. Valladolid. España.

1 Doctor en Medicina.

2 Licenciado en Medicina.

Correspondencia:

V.M. Asensio Sánchez

Hospital General Servicio Castellano-Leonés de Salud

Medina del Campo (Valladolid)

España

E-mail: victor_asensio@orangemail.es 
do, después está asintomático hasta el siguiente episodio. En la historia clínica no hay antecedentes familiares ni personales de interés. En la exploración la motilidad ocular es normal (fig. 1), aunque después de mirar fijamente a la izquierda desarrolla una exotropía de 20 grados (fig. 2), resolviéndose espontáneamente después de 50 segundos. El estudio de resonancia magnética nuclear orbitario y craneal fue normal. El test de edrophonium fue negativo. Se inició tratamiento con carmabacepina, primero con 200 mg y posteriormente con $400 \mathrm{mg}$ al día, mejorando los síntomas significativamente. Refirió, una disminución en un $50 \%$ en el número y la intensidad de los episodios al día, pero sin resolverse totalmente.

\section{DISCUSIÓN}

La neuromiotonía ocular es un proceso clínico raro caracterizado por la contracción de uno o varios músculos oculares, generalmente espontánea o en un intento de fijación y representa el fenotipo más severo de la hiperexcitabilidad de los nervios craneales oculares $(1,4)$. Descargas inapropiadas de las neuronas de los nervios motores oculares y/o inestabilidad de las membranas celulares pueden ser los responsables de la contracción. Aproximadamente el $40 \%$ de los pacientes con neuromiotonía adquirida presentan anticuerpos contra los canales de potasio $(1,4,5)$. Procesos como miastenia ocular, enfermedades desmielinizantes, forias descompensadas, enfermedad de Graves, espasmo de convergencia y mioquimia del oblicuo superior deben considerarse en el diagnóstico diferencial (5). En la mayoría de los casos descritos de NO hay historia de cirugía o más específicamente de radioterapia

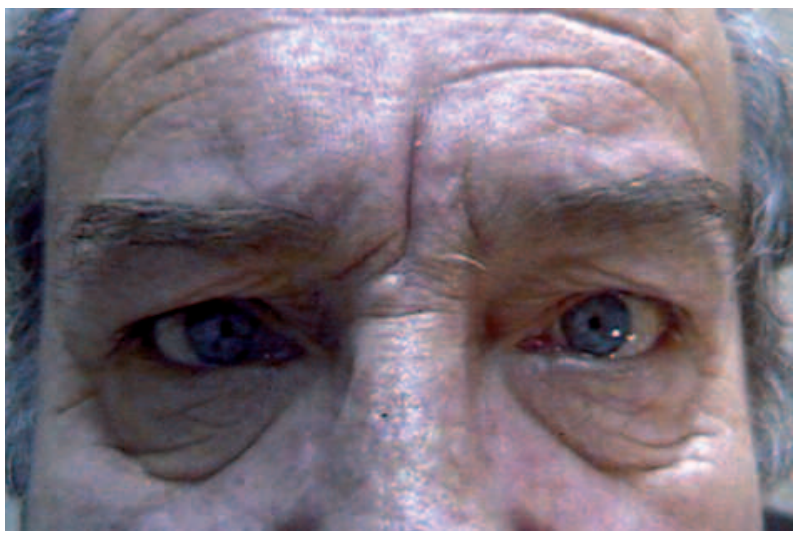

Fig. 1: Varón de 58 años con motilidad ocular normal.

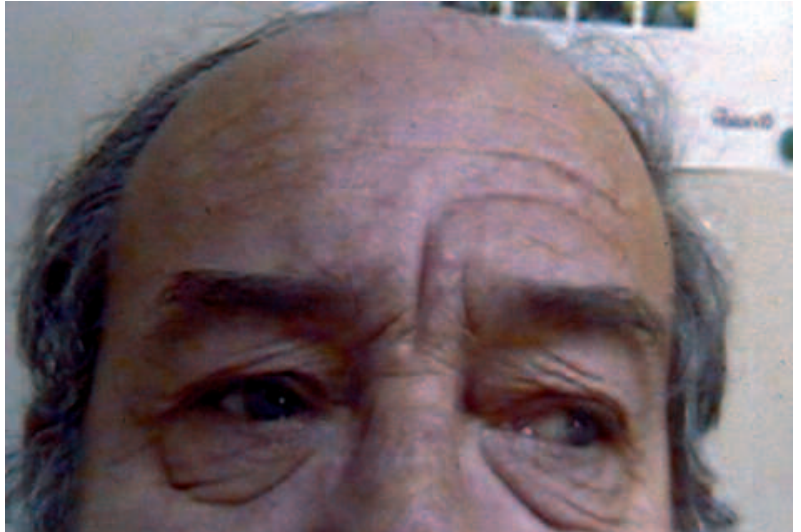

Fig. 2: Diplopía horizontal. Exotropía de unos 20 grados después de fijación prolongada a la izquierda. A los 50 segundos la motilidad retornó a la normalidad.

cerebral por tumores localizados en la región selar y/o paraselar o por lesiones compresivas en los motores oculares $(2,3,5)$. Cualquier nervio motor se puede afectar. La exploración oftalmológica en estos pacientes puede ser normal y sólo tras prolongados períodos de fijación o de mirada excéntrica se pueden poner de manifiesto los hallazgos característicos. En la literatura consultada sólo 6 casos son considerados como neuromiotonía ocular idiopática. En este trabajo se presenta un paciente de 58 años con neuromiotonía del sexto par craneal izquierdo, sin antecedentes de tratamiento radioterápico en el que no se encontró ninguna lesión, por lo que fue considerado como neuromiotonía ocular idiopática. Los pacientes con NO se benefician de fármacos como la carbamacepina que son estabilizadores de membrana (1-5), y todos deben ser estudiados para descartar una enfermedad intracraneal.

\section{BIBLIOGRAFÍA}

1. Frohman EM, Zee DS. Ocular neuromyotonia: clinical features, physiological mechanisms, and response to therapy. Ann Neurol 1995; 37: 620-626.

2. Hadjikoutis S, Morgan JE, Wild JM, Smith PE. Ocular complications of neurological therapy. Eur J Neurol 2005; 12: 499-507.

3. Tilikete C, Vial C, Niederlaender M, Bonnier PL, Vighetto A. Idiopathic ocular neuromyotonia: a neurovascular compression syndrome? J Neurol Neurosurg Psychiatry 2000; 69: 642-644.

4. Yee RD, Purvin VA, Azzarelli B, Nelson PB. Intermittent diplopia and strabismus caused by ocular neuromyotonia. Trans Am Ophthalmol Soc 1996; 94: 207-223.

5. Ezra E, Spalton D, Sanders MD, Graham EM, Plant GT. Ocular neuromyotonia. Br J Ophthalmol 1996; 80: 350-355. 